\title{
Perceptual Relearning of Complex Visual Motion after V1 Damage in Humans
}

\author{
Krystel R. Huxlin, ${ }^{1}$ Tim Martin, ${ }^{1}$ Kristin Kelly, ${ }^{1}$ Meghan Riley, ${ }^{1}$ Deborah I. Friedman, ${ }^{1,2}$ W. Scott Burgin, ${ }^{2}$ and \\ Mary Hayhoe ${ }^{3}$ \\ ${ }^{1}$ University of Rochester Eye Institute and ${ }^{2}$ Department of Neurology, University of Rochester, Rochester, New York 14642, and ${ }^{3}$ Department of Psychology, \\ University of Texas at Austin, Austin, Texas 78712-01871
}

\begin{abstract}
Damage to the adult, primary visual cortex (V1) causes severe visual impairment that was previously thought to be permanent, yet several visual pathways survive V1 damage, mediating residual, often unconscious functions known as "blindsight." Because some of these pathways normally mediate complex visual motion perception, we asked whether specific training in the blind field could improve not just simple but also complex visual motion discriminations in humans with long-standing V1 damage. Global direction discrimination training was administered to the blind field of five adults with unilateral cortical blindness. Training returned direction integration thresholds to normal at the trained locations. Although retinotopically localized to trained locations, training effects transferred to multiple stimulus and task conditions, improving the detection of luminance increments, contrast sensitivity for drifting gratings, and the extraction of motion signal from noise. Thus, perceptual relearning of complex visual motion processing is possible without an intact $\mathrm{V} 1$ but only when specific training is administered in the blind field. These findings indicate a much greater capacity for adult visual plasticity after V1 damage than previously thought. Most likely, basic mechanisms of visual learning must operate quite effectively in extrastriate visual cortex, providing new hope and direction for the development of principled rehabilitation strategies to treat visual deficits resulting from permanent visual cortical damage.
\end{abstract}

\section{Introduction}

In primates, visual information destined for conscious perception is sent from the eyes, through the dorsal lateral geniculate nuclei (dLGN) of the thalamus, to the primary visual cortex (V1). From there, visual information is distributed to and is processed by multiple extrastriate visual cortical areas (Felleman and Van Essen, 1991). Damage to extrastriate visual cortex causes relatively specific visual deficits, such as prosopagnosia after temporal cortex lesions (Damasio et al., 1982), motion perception abnormalities after lesions of parieto-occipital cortex (Zeki, 1991; Vaina, 1994), and color vision abnormalities after damage to the fusiform and lingual gyri (Vaina, 1994; Bartels and Zeki, 2000). In contrast and because it is the major gateway to the rest of the visual cortical system, V1 damage causes profound, homonymous losses in visual perception that affect most known visual modalities and are often referred to as "cortical blindness." Cortical blindness is severely disruptive to almost every aspect of the patient's daily life, affecting mobility, depth perception, reading, and driving (Pambakian and Kennard, 1997; Cole, 1999; Kerk-

Received 0ct. 9, 2008; revised Jan. 6, 2009; accepted Jan. 28, 2009.

This work was supported by an unrestricted grant from Research To Prevent Blindness (RPB) to the University of Rochester Eye Institute, by grants from the Pfeiffer Foundation (K.R.H., M.H.), the Schmitt Foundation (K.R.H., D.I.F., W.S.B., M.H.), and by National Institutes of Health Grants R01 EY05729 and RR09283 (M.H.). K.R.H. is an RPB Robert E. McCormick Scholar. We thank Terry Schaeffer and Dorothea Castillo for performing the Humphrey visual field tests. We also thank Jennifer Williams and Anasuya Das for their help in data collection and analysis.

Correspondence should be addressed to Dr. Krystel R. Huxlin, University of Rochester Eye Institute, 601 Elmwood Avenue, Box 314, Rochester, NY 14642. E-mail: huxlin@cvs.rochester.edu.

DOI:10.1523/JNEUROSCI.4882-08.2009

Copyright $\odot 2009$ Society for Neuroscience $\quad$ 0270-6474/09/293981-11\$15.00/0 hoff, 2000; Gutteridge and McDonald, 2004; McDonald et al., 2006).

Clinically, the approach to visual cortex damage is in dramatic contrast with the aggressive physical therapy administered to patients with damage to motor cortex (Hallett, 2001; Taub et al., 2002). Indeed, few treatment strategies are available to retrain vision after brain injury, and their history is controversial. Several detection perimetry systems have been developed (Zihl, 1981; Kasten et al., 1998), but their reported effectiveness has been confounded by claims of uncontrolled variables, such as light scatter and shifts in fixation affecting the patients' measured visual "recovery" (Balliet et al., 1985; Reinhard et al., 2005). As a result, these detection perimetry systems are not widely used clinically. However, even when eye movements are precisely monitored, intensive training of cortically blind subjects to discriminate a vertical sine-wave grating from a uniform background in their blind field can improve the patients' contrast sensitivity for this task (Sahraie et al., 2006). This suggests some capacity for perceptual learning in the blind field. Although these findings are promising, questions remain, particularly with regards to the retinotopic specificity, stimulus, and task specificities of training effects. Indeed, one concern is that the reason perceptual learning was elicited after V1 damage in the experiments of Sahraie et al. (2006) is that the spatiotemporal frequency content of stimuli was set at levels optimal for spatiotemporal channels known to be preserved after V1 damage (Barbur et al., 1994; Sahraie et al., 2003). One question our work addresses is whether perceptual learning is possible in the blind field of V1-damaged subjects 
when training stimuli are not limited to preserved spatiotemporal frequency channels.

The specificity of training effects is a critical issue, not only from a theoretical perspective but also from a practical viewpoint for the development of effective rehabilitation strategies. The present study probed further the properties of training-induced perceptual plasticity achievable in adult humans lacking an intact V1 by assessing the following: (1) the extent to which repetitive training in the blind field can improve both simple and complex motion perception; (2) the retinotopic specificity of traininginduced visual motion improvements in the blind field; and (3) whether training-induced visual motion improvements can generalize to stimuli and tasks other than those used during training.

\section{Materials and Methods \\ Subjects}

Seven adult subjects (for their demographics, see supplemental Table 1, available at www.jneurosci.org as supplemental material) were recruited 8-40 months after strokes that damaged V1 unilaterally, causing homonymous visual field defects. Inspection of structural head images [either magnetic resonance imaging (MRI) or computed tomography scans] verified the approximate extent and location of the damage, confirmed that V1 was affected, and ensured that the ipsilateral MT+ complex [containing both the middle temporal (MT) and medial superior temporal areas] and the contralateral hemispheres were intact (supplemental Figs. S1, S2, available at www.jneurosci.org as supplemental material). V1-damaged subject VC1 exhibited a stable, partial, right homonymous quadrant deficit 14 months after stroke. VC2 had a stable, partial, left homonymous hemianopia 8 months after stroke. VC3 had a stable, right homonymous hemianopia 20 months after stroke. VC4 exhibited a stable, partial left homonymous hemianopia 14 months after stroke. VC5 suffered from a partial, left homonymous hemianopia consequent on a stroke 40 months before enrollment in the study. VC6 had a right, homonymous hemianopia secondary to three strokes, the first 29 months and the last 12 months before the onset of testing. VC7 had a left homonymous hemianopia secondary to a burst aneurysm 12 months before recruitment in the study. All subjects possessed normal or corrected-tonormal vision when performing tests in this study. However, VC5 suffered from old (non-active) retinal degeneration in her right eye, affecting the macula. Given her unstable fixation with that eye, only data collected through her left eye were used for analysis. Her right eye was patched during all phases of testing and training. VC6 had also undergone cataract surgery bilaterally but was corrected with spectacles. All subjects were neurologically and cognitively intact except for their visual field defects. None exhibited visual or other forms of neglect, as determined through a thorough neurological and neuro-ophthalmological exam (supplemental Table 1, available at www.jneurosci.org as supplemental material). The experiments conducted as part of this study were reviewed and approved by the University of Rochester's Research Subjects Review Board. They adhered to the tenets of the Declaration of Helsinki and were conducted after obtaining the subjects' informed, written consent.

\section{Baseline measures of visual performance}

Perimetric visual field tests. Two sets of Humphrey (24-2 and 10-2) visual field tests were performed using a Humphrey Perimeter (HFA II 750) by the same ophthalmic technicians before the onset of visual retraining. This mapped the spatial extent of each subject's reported visual deficit and allowed us to verify its stability and to obtain a rough measure of the patient's fixation accuracy before the onset of training. The Humphrey eye tracker was turned on for all visual field tests except for VC3's right eye, for which it was not possible to obtain adequate tracking (because of abnormal dryness of the ocular surface) during this subject's initial visits. Adequate tracking was obtained for all subjects (including VC3) in posttraining Humphrey fields, performed again by the same technicians who performed the pretraining tests. The percentage of fixation losses, false positives, and false negative responses were computed for each visual field test.

Psychophysical measures of simple and complex motion sensitivity. After
Humphrey perimetry, each subject underwent psychophysical mapping of motion sensitivity. Subjects were positioned so that their eyes were exactly $42 \mathrm{~cm}$ from a 19 inch, NANAO computer monitor. A chin restforehead bar combination helped stabilize their heads. Their fixation was controlled by means of an ISCAN RK426 infrared camera monitoring both the pupil center and corneal reflection of the right eye. Real-time fixation information was fed into a Macintosh G4 computer (Apple Computers) running our custom psychophysics software. Limits were set so that, if subjects' eye moved by $>1^{\circ}$ from the fixation target during presentation of a visual stimulus, a loud tone resulted, and the trial was automatically aborted, recorded as a fixation break, and excluded from the analysis. Before each testing session, the ISCAN system was calibrated by requiring subjects to maintain fixation for $1000 \mathrm{~ms}$ within a small electronic window centered on a fixation target $0.1^{\circ}$ in diameter presented on the computer monitor in front of them. To accurately determine the offset and gain settings for each subject, the fixation target was then moved $10^{\circ}$ on either side of the center, along the vertical and horizontal meridians of the computer screen. Once fixation was calibrated, subjects were asked to perform direction discrimination and detection tasks centrally and then at different visual field locations to map their threshold performance for both simple and complex motion perception.

Direction discrimination task. Subjects were asked to discriminate the left-right direction of motion of either random-dot stimuli or luminance-modulated sine-wave gratings. They performed 50-100 trials of each task at different visual field locations, with each trial initiated by fixation of a small spot of light within an electronic window $2 \times 2^{\circ}$ in size. Steady fixation of this target for $1000 \mathrm{~ms}$ resulted in a tone signaling the onset of stimulus presentation during which subjects were required to maintain fixation. After $500 \mathrm{~ms}$, the stimulus and fixation spot disappeared, and the subjects were required to indicate whether they perceived the global direction of motion of the stimulus to be toward the right or the left by pressing the right or left arrow key on a computer keyboard placed in front of them. Correct and incorrect responses were signaled by different computer-generated tones, so that the subjects instantly knew whether they performed correctly or not. The sequence of presentation of rightward and leftward drifting stimuli was randomized. A break in fixation during stimulus presentation produced a loud, $1 \mathrm{~s}$ tone and the termination of the trial. To avoid positional biases, a correction procedure was used to detect any sequence of three incorrect responses to the same drift direction. When this occurred, the trial was repeated until the subject made a correct response. Data obtained during correction procedures were recorded but were not included in threshold calculations.

Visual stimuli. Random-dot stimuli in which the range of dot directions was varied (supplemental Fig. S3, available at www.jneurosci.org as supplemental material) were used to measure the subjects' range and motion signal thresholds for the left-right, global direction of motion of the whole stimulus (Huxlin and Pasternak, 2004). Random dots were presented at a density of 2.6 dots/degee ${ }^{2}$ within a circular aperture 2 and $2.5^{\circ}$ in diameter for the blind spot testing in control subjects $\mathrm{C} 1$ and $\mathrm{C} 2$, $6^{\circ}$ in diameter for $\mathrm{VC} 1,8^{\circ}$ in diameter for $\mathrm{VC} 2, \mathrm{VC} 4$, and $\mathrm{VC} 5$, and $12^{\circ}$ in diameter for VC3. The dots in the random-dot stimuli were $0.06^{\circ}$ in diameter. They were repeatedly displaced in a direction chosen randomly from a uniform distribution, with a new set generated on each frame. The dots moved at a speed of 10 or $20 \% \mathrm{~s}\left(\Delta t=13 \mathrm{~ms} ; \Delta x=0.13\right.$ or $0.26^{\circ}$, respectively) for a lifetime of $250 \mathrm{~ms}$ (half the stimulus duration). Dot brightness was $33 \mathrm{~cd} / \mathrm{m}^{2}$, and background luminance was $45 \mathrm{~cd} / \mathrm{m}^{2}$, which minimized sensitivity to changes in light scattering from the stimulus during its presentation. Contrast was reversed for part of the blind spot testing of control subjects $\mathrm{C} 1$ and $\mathrm{C} 2$, with dot and background brightness set at 45 and $3 \mathrm{~cd} / \mathrm{m}^{2}$, respectively. By presenting bright dots on a dark background, sensitivity to light scatter generated by the stimulus was maximized, allowing us to assess whether such information could be at all used to extract a global directional signal sufficient to perform the task.

Vertical, drifting sine-wave gratings were used to measure contrast sensitivity for simple (local) direction discrimination. They were presented in a circular aperture $8^{\circ}$ in diameter and drifted either to the left or the right within this aperture. The mean display luminance for this task was $20 \mathrm{~cd} / \mathrm{m}^{2}$, and stimulus duration exhibited a $250 \mathrm{~ms}$ raised cosine 
temporal envelope. Contrast thresholds were measured while subjects discriminated the left-right direction of motion of the stimulus by varying grating contrast, computed as $\left(L_{\min }-L_{\max } / L_{\min }+L_{\max }\right) \times 100$, where $L_{\min }$ and $L_{\max }$ refer to minimal and maximal luminance in the display.

Threshold measurement. A staircase procedure was used to measure contrast, motion signal, and direction range thresholds. During each session, the stimulus set was varied along a selected dimension (luminance contrast for sine-wave gratings, percentage of signal dots, and range of dot directions for random-dot stimuli), from the easiest to the most difficult. Three consecutive correct responses caused an increase in stimulus difficulty (i.e., reduced contrast, decreased proportion of signal dots, or increased range of dot directions), whereas a single incorrect response decreased it. The staircase used for contrast measurements was $100,63,44.4,31.3,22,15.5,10.9,7.7,5.4,3.8,2.7,1.9,1.3,0.94,0.67,0.46$, $0.33,0.23$, and $0.16 \%$ contrast. The staircase used for direction range threshold measurements was $0,40,80,120,160,200,240,280,320$, and $355^{\circ}$ range. The staircase used for motion signal measurements was 100 , $71.4,51,36.4,26,18.6,13.3$, and $0 \%$ of signal dots. Thresholds were calculated by fitting a Weibull function to the percentage correct performance at each stimulus level and computing the stimulus value (luminance contrast, direction range, or percentage signal), resulting in $75 \%$ correct performance. This criterion was selected because it lay halfway between chance (50\% correct) and $100 \%$ correct on this two-alternative task. Threshold luminance contrast was further converted to contrast sensitivity by calculating its inverse and multiplying it by 100 .

\section{Visual retraining}

After completing baseline testing, a computerized visual retraining system was set up in each subject's home. A chin-forehead-rest system allowed the subjects' eyes to be positioned $42 \mathrm{~cm}$ away from the computer monitor presenting the visual stimuli. The training stimulus settings were based on optimal performance attained during baseline psychophysical testing: $\mathrm{VC1}$ trained with a stimulus $6^{\circ}$ in diameter and dots traveling at $10^{\circ} / \mathrm{s}$, VC2, VC4, and VC5 trained with an $8^{\circ}$ stimulus and dots traveling at $20 \%$ s; and VC3 trained with a $12^{\circ}$ stimulus and dots traveling at $10 \%$ s. Different stimulus sizes were used for the different subjects because of their differing blind field topographies. Stimuli were chosen to maximize the area of visual field retrained, while at the same time being able to fit completely inside most areas of the blind field. Subjects performed one 300-trial training session per day and saved their data to disks that were mailed weekly to our laboratory. Training of the blind field started at locations close to the border with the intact hemifield, moving progressively deeper into the blind field as direction range thresholds improved at each trained location. Training at each site continued until the subject performed with at least $75-80 \%$ accuracy and attained direction range thresholds within the normal range.

\section{Posttraining visual evaluations}

After 9-18 months of visual training at home, patients were brought back to the laboratory for follow-up evaluations in which their Humphrey visual fields and psychophysical performance were remeasured as described for the baseline testing above. In addition, we also measured three of the retrained patients' (VC1, VC3, and VC4) eye movements and scan paths during performance of the right-left global direction discrimination task at a retrained location in their blind field (specific locations tested were those shown in Fig. 1A). This was done using a headmounted ASL Mobile Eye (Applied Science Laboratories), which tracks the right pupil with a resolution of $0.1^{\circ}$ and superimposes its position on a real-time $30 \mathrm{~Hz}$ video record of the patients' field of view. In this case, the patient's field of view included the computer monitor presenting the fixation spot and stimulus to be discriminated. The Mobile Eye was first calibrated using a grid of nine spots presented on the computer monitor, and the three trained subjects were asked to perform 50 trials of the global direction discrimination task at one of their retrained blind field locations. The Mobile Eye video record was then analyzed frame by frame. The eye position relative to the fixation spot was plotted separately for each correct and incorrect trial, during both the $330 \mathrm{~ms}$ before each stimulus appearance (while the subjects were supposed to look at the fixation spot, in the absence of a stimulus) and stimulus presentation (500 ms).

\section{Statistical methods}

For direction range, motion signal, and contrast thresholds, the mean and SD of the parameters of interest were compared between locations in the intact and blind fields, as well as a function of training, with paired Student's $t$ tests. All inferential tests were two tailed, and $p<0.05$ was considered statistically significant.

To assess change across time in the 24-2 Humphrey visual field perimetry data, we used $k$-means cluster analysis to divide the visual field locations assessed. $k$-means cluster analysis groups multiple cases by maximizing the between-group variance but minimizing the withingroup variance (Duda and Hart, 1973). For multiple variables (i.e., repeated tests), this results in groups defined by patterns across the variables (i.e., across the repeated tests). Visual field locations with normal or near-normal initial sensitivity (such as in the intact hemifield of vision) would be expected to remain unchanged. Likewise, many locations that were abnormally insensitive (such as those in untrained portions of the blind field) would remain so after training. For training-induced improvements in the visual field, we would expect a subset of locations to show abnormally low sensitivity before training but a gain of some degrees of sensitivity after training. Therefore, we separated the visual field results for each participant into three clusters: high sensitivity initially + no change after training, low sensitivity initially + no change after training, and low sensitivity initially + significant change after training. If our hypothesis is incorrect and there is no third group of visual field locations that improves over time, then we would expect the third cluster to reflect only random variability or a different pattern for which we have no a priori hypotheses, as was the case for VC7.

For posttraining data collected with the ASL Mobile Eye, four variables of eye position were analyzed from the video record in a frame-by-frame manner. These included horizontal and vertical coordinates of eye position relative to the center of the fixation spot (in degrees of visual angle) in two epochs: during the $330 \mathrm{~ms}$ before stimulus onset and the $500 \mathrm{~ms}$ of stimulus presentation. Because no saccades and no eye movements $>2^{\circ}$ were ever observed in these epochs, we computed a single, average eye position for each epoch, trial, and subject. Independent-sample $t$ tests were then used to assess differences between correct and incorrect trials, during the $330 \mathrm{~ms}$ prestimulus and the $500 \mathrm{~ms}$ stimulus conditions, at the individual subject level. Again, $p<0.05$ was considered statistically significant.

\section{Results}

\section{V1 damage impairs both simple and complex}

\section{motion perception}

Contrast sensitivity for the left-right direction discrimination of drifting, luminance-modulated sine-wave gratings was severely reduced in the blind field of VC1-VC7 (15 \pm 5.4 in the bad field vs $121 \pm 24$ in the intact hemifield; $p=0.002$, paired Student's $t$ test). Complex motion processing was also severely affected in the blind field. None of the subjects were able to discriminate the global direction of motion of random-dot stimuli in which the range of dot directions was $>9 \pm 9^{\circ}$ around the right or leftward vector (compared with $309 \pm 4.5^{\circ}$ in their intact hemifield; a significant difference, $p=1.2 \times 10^{-7}$, paired Student's $t$ test). Similarly, random-dot stimuli presented in the blind field had to contain an average of $70 \pm 12 \%$ signal dots as opposed to $15 \pm$ $3 \%$ signal dots in the intact hemifield for subjects with V1 damage to correctly discriminate the global direction motion of the stimulus at a rate of $75 \%$ correct or better. The difference between motion signal thresholds in the intact and blind hemifields was also statistically significant ( $p=0.014$, paired Student's $t$ test). Thus, all measured aspects of motion perception, whether simple or complex, were impaired in the blind field. However, the subjects' fixation was very precise. Measurements collected with the ISCAN tracker showed that the average SD of the subjects' hori- 

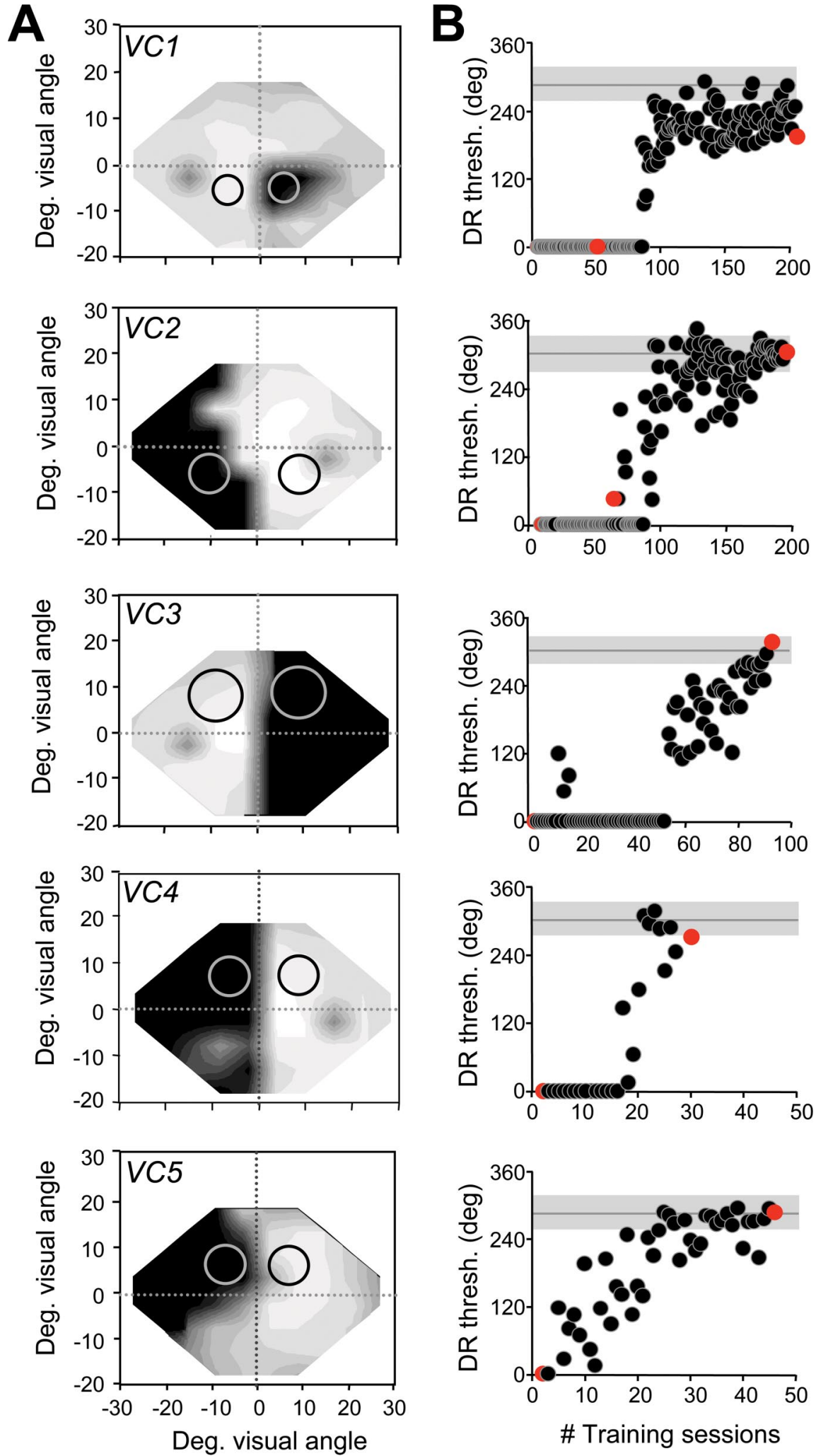

Figure 1. Training-induced improvements in direction range thresholds in the blind fields of VC1-VC5. A, Size and location of random-dot stimuli on which subjects were first trained in their blind field indicated on composite Humphrey visual field maps (for grayscale, see supplemental Fig. 1, available at www.jneurosci.org as supplemental material). (ircles illustrate the sizes and locations of random-dot stimuli used to measure performance at equivalent locations in the intact and blind hemifields. $\boldsymbol{B}$, Scatter plots of direction range (DR) threshold (thresh.) performance as a function of the number (\#) of training sessions administered at blind field locations in $\boldsymbol{A}$. Black dots represent data collected by the subjects during in-home training. Red dots represent data collected from the same subjects in the laboratory, where central fixation was controlled using an integrated ISCAN system. Note the close correlation between the subjects' in-home performance and that recorded in the laboratory. Note also the variability in the number of training sessions, each consisting of 300 trials, required for different subjects' direction range thresholds to reach zontal eye position around the fixation spot was $0.62 \pm 0.16^{\circ}$ for a period of $\sim 10 \mathrm{~s}$. In comparison, three control (visually intact) subjects, who were extremely experienced at doing visual psychophysics with controlled fixation, had an average SD of fixation around the same target and over the same time period of $0.47 \pm 0.21^{\circ}$. There was no statistically significant difference between the V1-damaged and control subjects in terms of fixation accuracy ( $p=$ 0.599 , two-tailed Student's $t$ test). The stability of our patients' fixation during psychophysical testing was further supported by the fact that, of an average \pm SEM of $1197 \pm 101$ trials performed in the blind field during baseline testing, only $7.4 \pm$ $2.2 \%$ of these trials contained a fixation break detected by the ISCAN tracker.

Repetitive training improves direction integration in the blind field

After baseline visual testing, VC1-VC5 underwent daily visual training sessions at home, whereas VC6 and VC7 did not undergo visual training over a similar period of time ( $>1$ year). For training subjects, a training session consisted of performing 300 trials (lasting $\sim 30 \mathrm{~min}$ ) of a twoalternative, forced-choice, left-right direction discrimination task using randomdot stimuli in which the range of dot directions was varied in a staircase procedure (supplemental Fig. S3, available at www.jneurosci.org as supplemental material).

Over the first $10 \mathrm{~d}$ of in-home training, direction range thresholds at the blind field locations selected (Fig. $1 A$, light gray circles) averaged $13 \pm 13^{\circ}$ (Figs. 1, 2A). This very abnormal, mean direction range threshold was not significantly different from that measured in the laboratory (i.e., with controlled fixation). Training gradually increased direction range thresholds in each subject until they stabilized at $\sim 293 \pm 12^{\circ}$, a value well within the normal range for this task (Figs. $1 B, 2 A, B$ ). Psychometric functions for this task were almost flat before training but became normal after training (Fig. $2 \mathrm{~B}$ ). Between 20 and 100 training sessions (i.e., 600030,000 trials) were required for recovery of direction range thresholds in the blind field. This demonstrates significant inter-

\footnotetext{
$\leftarrow$

and stabilize within the normal range. This normal range was represented by the mean (gray horizontal line) and SD of the mean (gray shaded area around the gray line) direction range thresholds obtained at the "control" locations in the intact hemifield of each subject (black circles in $\boldsymbol{A}$ ). Deg., Degree.
} 


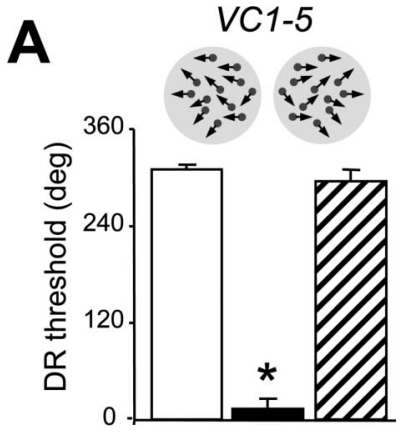

B
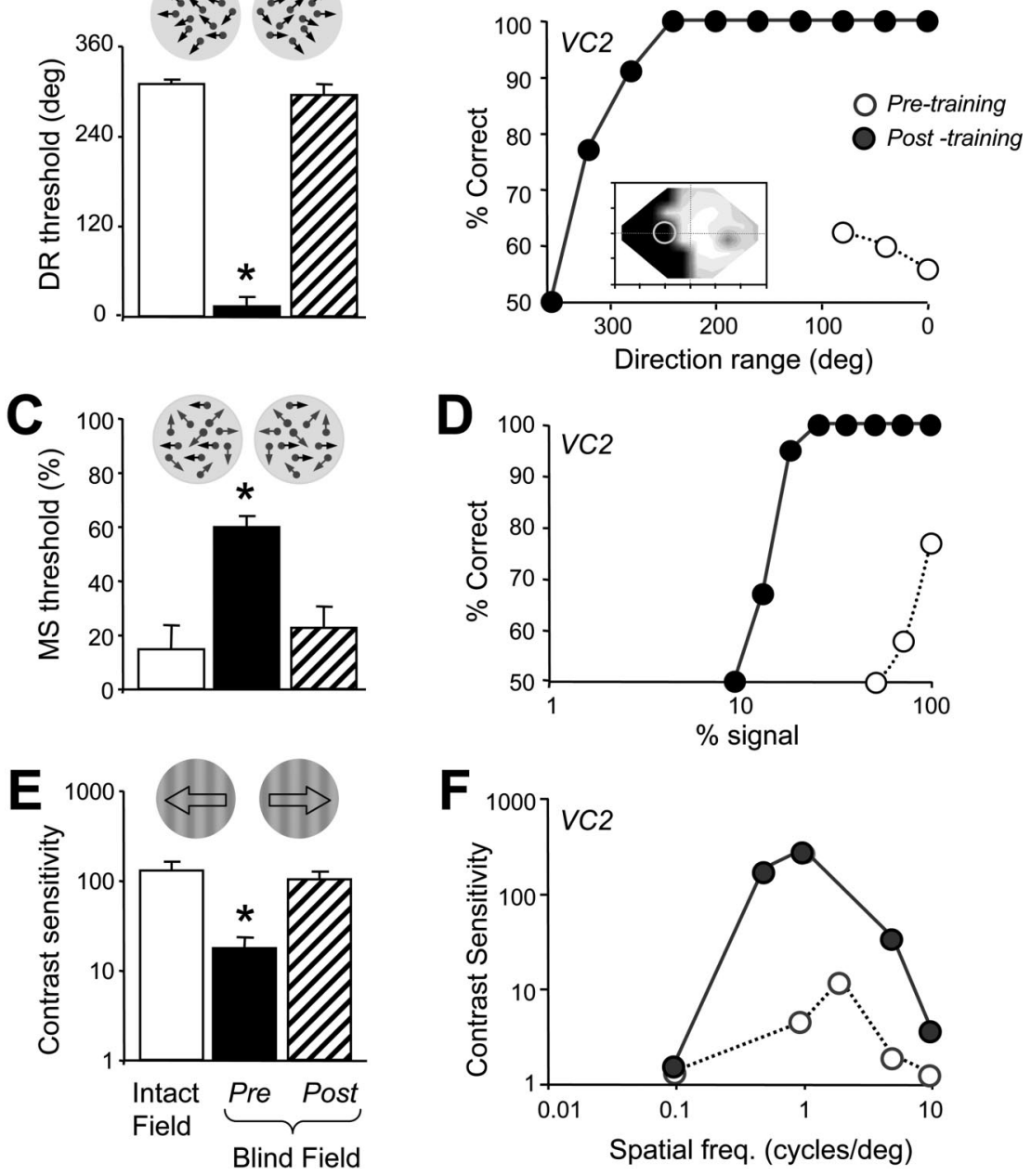

Figure 2. Global motion discrimination training improves contrast sensitivity and the extraction of motion signal from noise. $\boldsymbol{A}$, Histogram illustrating how global direction discrimination training returns direction range (DR) thresholds to normal (relative to those in the intact hemifield; $p=0.127$, paired Student's $t$ test) at the retrained blind field locations in VC1-VC5. B, Sample psychometric function for VC2 performing a left-right, global direction discrimination task in her blind field (see location circled in the Humphrey visual field map inset). Before training (open circles), percentage correct performance hovered just above chance at the lowest direction range levels. After training (filled circles), performance improved dramatically and a normal psychometric function was attained for this task. $\boldsymbol{C}$, Histogram illustrating the positive effect of global direction discrimination training on motion signal (MS) thresholds in the blind field of VC1-VC5. Training on random-dot stimuli with a large range of dot directions (not motion signal) recovered the subjects' ability to extract motion signal from random directional noise at the trained locations. After training, motion signal thresholds were not significantly different ( $p=0.759$, paired Student's $t$ test) from those in the intact hemified (white column). D, Sample psychometric function for VC2 discriminating the left-right direction of motion of random-dot stimuli in her blind field (see location circled in the Humphrey visual field map inset in $\boldsymbol{B}$ ). The percentage of noise dots was varied throughout the session, but, before training (open circles), only stimuli with $100 \%$ signal dots could be discriminated at a $75 \%$ correct level. After training on direction range thresholds, however, performance and the psychometric function returned to normal (filled circles). $\boldsymbol{E}$, Histogram illustrating the effect global direction discrimination training using random dots (of set contrast) on contrast sensitivity for left-right direction discrimination of luminance-modulated, drifting sine-wave gratings (spatial frequency, 0.5 or 1 cycle/\% temporal frequency, $10 \mathrm{~Hz}$ ). Training with random dots significantly improved contrast sensitivity at the retrained, blind field locations ( $p=0.405$, paired Student's $t$ test, relative to equivalent locations in the intact hemifields). $\boldsymbol{F}$, Plot of contrast sensitivity versus spatial frequency for VC2 before (open circles) and after (filled circles) training with random-dot stimuli at the location indicated in $\boldsymbol{B}$. Temporal frequency was held at $10 \mathrm{~Hz}$. Global direction discrimination training significantly improved contrast sensitivity at the trained, blind field location, with the greatest improvements occurring between 0.5 and 2 cycles $/^{\circ}$. All data in the histograms are expressed as means and SEM. ${ }^{*} p<0.05$, paired Student's $t$ tests relative to intact hemifield values. Deg., Degree; freq., frequency.

individual variability in the rate of relearning. Control V1damaged subjects VC6 and VC7 showed no significant change in direction range thresholds within their blind field over a period of $>500 \mathrm{~d}$ (supplemental Fig. S2 D, available at www.jneurosci.org as supplemental material).
Eye movements and light scatter do not explain training-induced improvements in direction range thresholds in the blind field

Much of the controversy surrounding some previous attempts to rehabilitate vision after V1 damage revolved around the lack of appropriate eye movement control and the potentially confounding effects of light scatter (Bach-y-Rita, 1983; Balliet et al., 1985; Reinhard et al., 2005). In the present study, just as in the study of Sahraie et al. (2006), eye movements were not tracked during in-home training. They were only tracked during psychophysical tests in the laboratory, which were conducted before, during, and after training (Fig. $1 B$, red data points). An integrated ISCAN RK426 pupillometer was used to track movements of the right eye during all psychophysical tests and abort all trials in which fixation deviated by $>1^{\circ}$ from the fixation spot during stimulus presentation. Only data for which similar thresholds were obtained during training at home and in the laboratory are presented here. After training, of $1132 \pm 61$ trials performed in the subjects' blind field with ISCAN eye tracking, only $6.9 \pm 2.4 \%$ of these trials were aborted as a result of a fixation break. This was not statistically different from the proportion of fixation breaks observed before training (7.4 \pm $2.2 \%$ ) under identical testing circumstances.

However, it is conceivable that, during in-home training, subjects learned to make microsaccades or bias their eye position toward their blind field. If this were the case, then we should see a bias in the distribution of eye positions toward the blind field, particularly on correct trials and immediately on stimulus appearance. To test for this possibility, right eye position traces were also recorded and analyzed relative to both the fixation spot and the visual stimuli to be discriminated using an ASL Mobile Eye during posttraining tests in VC1, VC3, and VC4. These measurements confirmed that the improved direction range thresholds in these subjects were not attributable to increased microsaccades or other changes in eye movements or position toward visual stimuli in their blind field (supplemental Fig. S4, available at www.jneurosci.org as supplemental material). For VC1, there were no detectable differences in their horizontal or vertical eye position relative to the fixation spot between correct and incorrect trials during either the $330 \mathrm{~ms}$ before stimulus onset $\left(t_{(46)}=-0.799, p=0.428\right.$ and $t_{(46)}$ $=0.495, p=0.623$, respectively) or the stimulus presentation in the blind field $\left(t_{(45)}=-0.021, p=0.983\right.$ and $t_{(45)}=0.635, p=$ 0.528 , respectively). Likewise, for $\mathrm{VC} 3$, there were no significant 
differences between correct and incorrect trials in either horizontal or vertical gaze coordinates before stimulation $\left(t_{(48)}=\right.$ 1.988, $p=0.053$ and $t_{(48)}=1.547, p=$ 0.128 , respectively) or during stimulation $\left(t_{(48)}=0.631, p=0.531\right.$ and $t_{(48)}=0.578$, $p=0.566$, respectively). Finally, for VC4, there was no significant difference between correct and incorrect trials before stimulation in the horizontal or vertical direction $\left(t_{(48)}=-1.569, p=0.123\right.$ and $t_{(48)}$ $=-0.843, p=0.403$, respectively) or during stimulation $\left(t_{(48)}=1.778, p=0.082\right.$ and $t_{(48)}=-0.199, p=0.843$, respectively). Thus, analysis of eye movement traces during fixation using the Mobile Eye after training revealed mean jitter magnitude from the fixation spot of $<1^{\circ}$ on each recorded trial and no significant difference in mean eye deviation from fixation between correct and incorrect trials, suggesting that subjects were not attaining correct responses by shifting their eyes toward the stimulus in their blind field.

Nevertheless, by tracking only the right eye during psychophysical testing, it is conceivable that we may have missed independent movements of the left eye toward the stimulus in all patients. That such eye movements could explain the observed improvements in motion integration is unlikely because of the following: (1) movements would have had to be fairly large $\left(4-12^{\circ}\right.$ in size $)$ to reach the nearest portion of some of the random-dot stimuli to be discriminated, (2) all patients would have to independently learn to move only the left eye during training at home, when eye position was not monitored, and (3) improvements in direction range thresholds were accompanied by matching improvements in Humphrey visual field performance in which eye tracking was performed monocularly for each eye (see below for effect of training on Humphrey visual fields). If improved direction range thresholds were attributable to the subjects learning to independently move their left eye toward the blind field, monocular tracking during Humphrey perimetry should have eliminated improvements on this test. It did not.

Finally, to test whether the random-dot stimuli used in the present study could generate sufficient light scatter to perform global motion integration, we recruited two visually intact controls ( $\mathrm{C} 1$ and $\mathrm{C} 2)$ and patched each of their eyes in turn. With one eye patched, a random-dot stimulus $2-2.5^{\circ}$ in diameter, consisting of dots moving with $0^{\circ}$ range and $100 \%$ motion signal but otherwise identical to that used in our cortically blind patients, was placed within the confines of the blind spot (supplemental Fig. S5B, available at www.jneurosci.org as supplemental material). The control subjects were asked to report on the left or rightward global direction of motion of the stimulus. Despite their ability to reliably detect stimulus onsets and offsets, neither subject was able to perform this task above chance (50\% correct). This was still the case when contrast was reversed and bright random dots were presented on a black background, maximizing
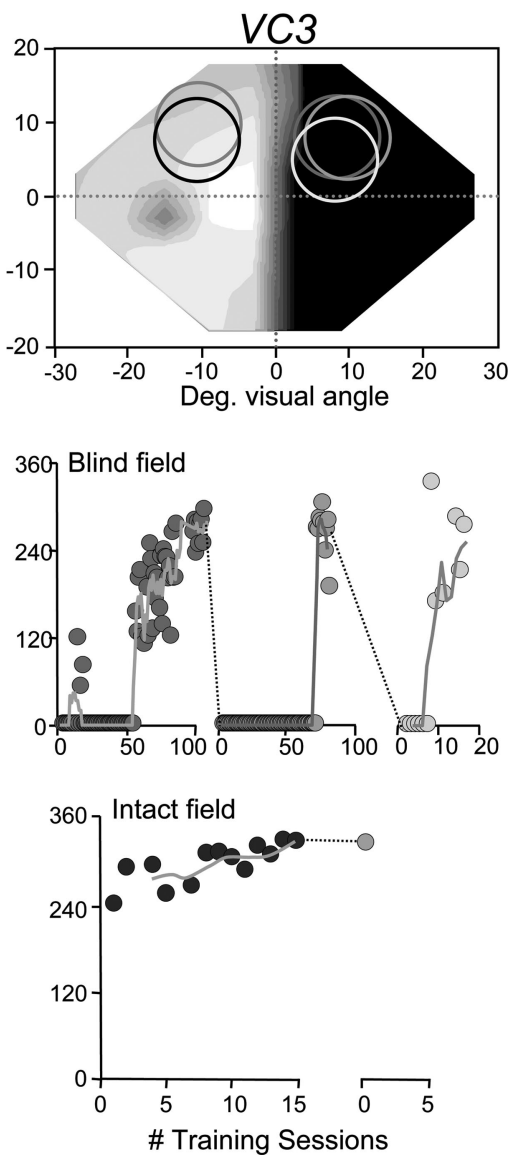

Figure 3. Retinotopic specificity of training-induced improvements in direction range thresholds. $\boldsymbol{A}$, Visual field maps for VC2 and VC3, illustrating the locations where visual training was performed. The shade of gray of circles in the visual fields match the

the amount of light scatter falling onto photosensitive portions of the retina around the optic disc. C1 was also repeatedly trained to discriminate global direction of motion in her left eye's blind spot over 17 consecutive days (5100 trials). Training caused no improvement in either percentage correct or direction range thresholds (supplemental Fig. S5B, available at www.jneurosci.org as supplemental material). Thus, light scatter falling on intact visual circuitry makes stimulus onsets and offsets detectable. However, it provides insufficient information for an intact (let alone a damaged) visual system to learn to compute global direction of motion, at least with stimulus parameters (dot size, density, contrast, speed) used in the present study.

\section{Retinotopic specificity of training-induced improvements}

Once direction range thresholds had recovered to normal levels at the first blind field location trained, the training stimulus was moved to other locations in the blind field to assess the retinotopic extent of visual relearning (Fig. 3). Just as in our previous animal studies (Huxlin and Pasternak, 2004; Huxlin et al., 2008), training-induced perceptual improvements in humans were restricted to retrained visual field locations. Whenever the stimulus was moved to a new blind field location, whose center was $>2-4^{\circ}$ 
away from the center of the retrained location, direction range thresholds dropped to $0^{\circ}$ (Fig. $3 B, C$ ). By mapping the confines of the training stimulus with smaller (but otherwise identical) random-dot stimuli, we ascertained that subjects needed only the most proximal $2-4^{\circ}$ of the training stimulus to perform the task (supplemental Fig. S5A, available at www.jneurosci.org as supplemental material). However, retraining was possible at each location tested, even when the edge of the training stimulus closest to the midline was $>10^{\circ}$ away from intact portions of the subject's visual field (Fig. $3 A, B$ ).

This experiment allowed us to note a variable rate of improvement in direction range thresholds between subjects but also between blind field locations in a given subject. No significant correlations could be drawn between learning rate, stimulus eccentricity, stimulus depth within the blind field, practice at the task, age of the patient, or age of the brain damage. Most likely, the variable learning rate was caused by uneven damage to the circuitry underlying vision in different regions of the visual field within a given subject as well as between subjects.

\section{Learning in the blind hemifield differs from learning in the intact hemifield}

When first measured in the intact hemifields of VC1-VC5, direction range thresholds averaged $297 \pm 34^{\circ}$ (Fig. 3C). After a few training sessions $(4-15)$, their thresholds improved by between 30 and $90^{\circ}$ of range. In contrast to the tight retinotopic specificity of training-induced improvements in their blind field, however, improvements in global motion thresholds, although small, transferred effectively to adjacent locations in the subject's intact, visual hemifield (Fig. 3C).

\section{Global motion training improves visual sensitivity and the ability to extract motion signal from noise in the blind field} An important aspect of visual training is the degree to which it generalizes to modalities and tasks not specifically trained. Nine to 18 months after the initial baseline threshold measurements, all subjects were brought back into the laboratory for posttesting. Consistent with their in-home performance and in contrast with their initial, pretraining thresholds, VC1-VC5 now exhibited normal psychometric functions and normal direction range thresholds at the retrained locations (Fig. 2A,B). However, motion signal thresholds (Fig. 2C,D) and contrast sensitivity for direction (Fig. 2E, $F$ ) were also significantly improved at the trained, blind field locations. For contrast sensitivity, the spatial and temporal frequencies at which the greatest posttraining improvements were attained hovered around $0.5-1 \mathrm{cycles} /{ }^{\circ}$ and 10 $\mathrm{Hz}$ (Fig. $2 \mathrm{~F}$ ), values matching the known magnitude of the spatiotemporal channels thought to mediate aspects of blind sight (Barbur et al., 1994; Sahraie et al., 2003, 2006).

Global direction discrimination training also increased sensitivity for the detection of static spots of light, as indicated by decreases in the size of the regions with $<5 \mathrm{~dB}$ of sensitivity on our trained subjects' Humphrey visual fields and a remarkable stability of Humphrey field defects in VC6 and VC7 (Fig. 4). On average, trained subjects attained $13.5 \pm 5 \mathrm{~dB}$ ( 1 to 2 log units) of improvement per eye, which occurred primarily within and around the retrained visual field locations. $k$-means cluster analysis (Duda and Hart, 1973) revealed significant improvements in a subset of visual field locations retrained in VC1-VC5, whereas no significant improvements were measured in VC6 and VC7 (Fig. $4 B$ ). Because the number of fixation losses, false positives and false negatives remained low or changed only minimally be- tween pretesting and posttraining tests, improvements in Humphrey visual fields were most likely related to training.

\section{Global motion training increases awareness of the stimulus in the blind field}

An important issue when measuring perceptual thresholds during retraining is whether patients are aware of their improvements. It would certainly be conceivable for those who exhibit some form of blind sight to remain unaware of improvements, as was shown by Sahraie et al. (2006). Indeed, neuronal networks in the dorsal visual pathway, which should be optimally stimulated by our retraining paradigm, are specialized for visuomotor control rather than conscious perception (Goodale, 1998). Contrary to this expectation, all subjects in the present study reported increasing conscious awareness of the visual stimulus in their blind field as their direction range thresholds improved. At the start of training, subjects reported "sensing" that a visual stimulus appeared and disappeared, without being able tell that it was moving or that it was made of dots. As training progressed, a sensation of motion was eventually felt, followed by an improvement in the ability to correctly extract global direction of motion from the stimulus. This was coincident with the patients' first report of actively seeing a proportion of the random dots. This proportion gradually increased as direction range thresholds reached nearnormal levels. Thus, awareness of training stimuli grew stronger and more complex as training progressed, paralleling improvements in integration thresholds.

\section{Discussion}

Cortical blindness is a devastating loss of visual perception that, in primates, results from damage to $\mathrm{V} 1$ or its immediate inputs. Although the contribution of V1 to visual sensation has been extensively studied, its role in perceptual learning is less well defined. In an intact visual system, visual learning often exhibits specificity for fundamental stimulus features, such as orientation (Ramachandran and Braddick, 1973; Fiorentini and Berardi, 1980; Crist et al., 1997), spatial frequency (Fiorentini and Berardi, 1980), direction of motion (Ball and Sekuler, 1987; Vaina et al., 1995), visual field location (Fiorentini and Berardi, 1980; Karni and Sagi, 1991; Shiu and Pashler, 1992; Crist et al., 1997), and eye of presentation (Karni and Sagi, 1993). These specificities are consistent with the smaller receptive field sizes, selectivity for stimulus attributes and ocular dominance observed in early visual cortical circuits, including V1 (Maunsell and Newsome, 1987). A potentially important role for V1 in visual learning is further supported by electrophysiological studies in monkeys (Crist et al., 2001; Schoups et al., 2001), functional MRI (Schwartz et al., 2002; Furmanski et al., 2004; Walker et al., 2005), and EEG (Pourtois et al., 2008) studies in humans, which have demonstrated functional plasticity in V1 to parallel behaviorally measured visual learning. Thus, if V1 is important for visual learning, can such learning still occur in an adult visual system in which V1 has been permanently damaged and in which perception is clearly deficient? As mentioned previously, a few studies have suggested that training-induced improvements in simple visual sensitivity may be possible after V1 damage (Sahraie et al., 2006; Raninen et al., 2007). However, the true extent and properties of training-induced perceptual plasticity achievable in an adult visual system lacking an intact V1 remain to be elucidated.

The present study demonstrates for the first time the effectiveness of repetitive training for improving both simple and more complex visual motion processing in the blind field of V1damaged human adults. Improving visual motion processing af- 

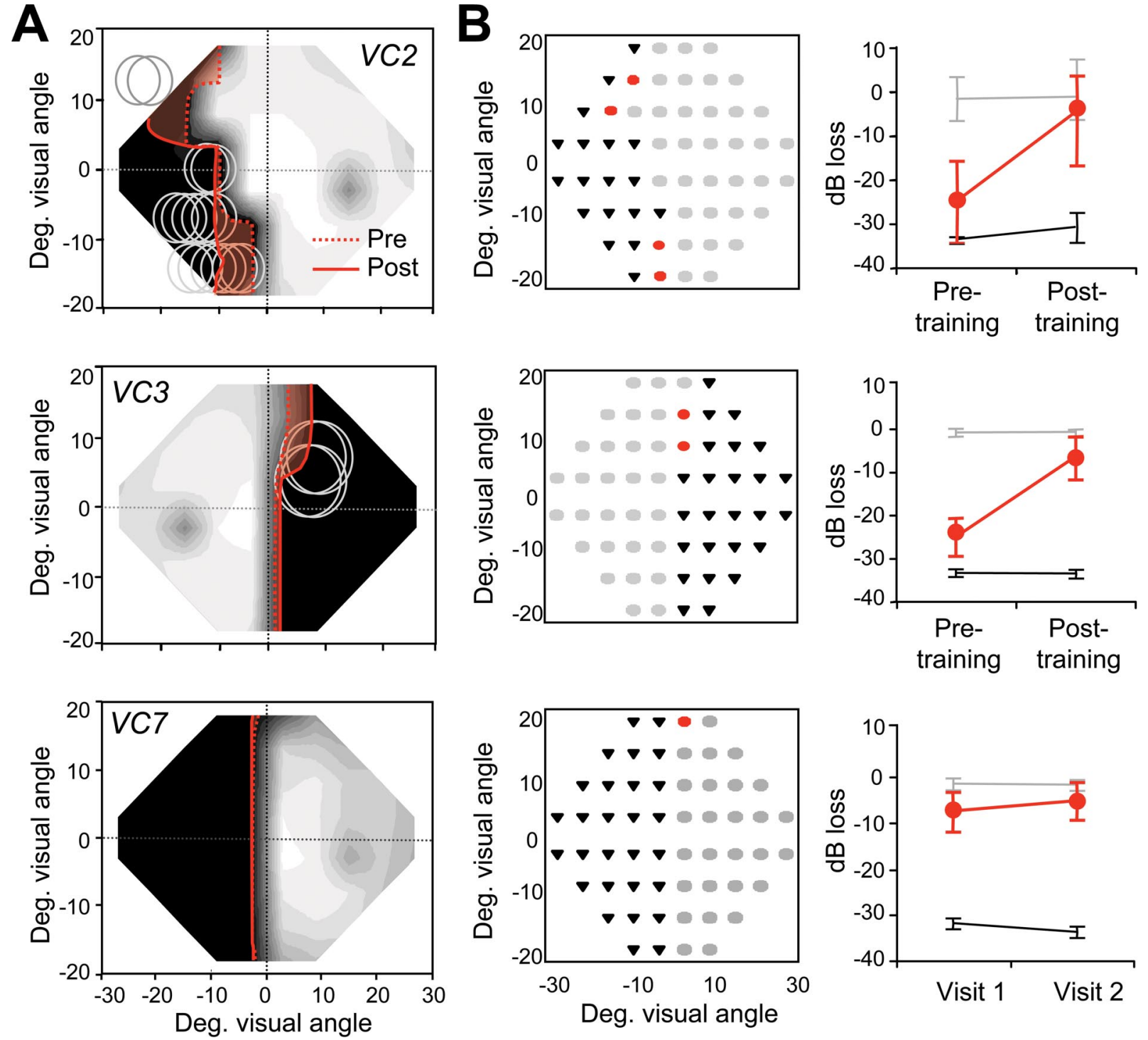

Cluster 1 Cluster 2

Cluster 3

Figure 4. Global motion discrimination training decreases the size of Humphrey visual field defects. $\boldsymbol{A}$, Composite Humphrey visual maps obtained in VC2 and VC3 after global direction discrimination training at locations denoted by gray circles and, in VC7, more than 1 year after his initial examination. The solid red lines outline the $5 \mathrm{~dB}$ contour line after training or during visit 2 for VC7. Note that for VC2 and VC3 (but not for VC7), this border had receded significantly (shaded red area) from the $5 \mathrm{~dB}$ pretraining border (dotted red line), although the posttraining Humphrey maps were obtained 30 or more months after stroke. Color conventions and scaling for the composite maps are as in Figure 1 and supplemental Figure S1 (available at www.jneurosci.org as supplemental material). Enlargements of the visible field always overlapped with retrained locations. B, Cluster analysis of Humphrey visual fields for VC2, VC3, and VC7, illustrating the change in mean pattern deviation for different field locations over time. The left graph in each pair color codes the visual field locations assigned to each cluster. The right graph then illustrates the mean pattern deviation (in decibels) for each cluster across time. Light gray (Cluster 1) represents visual field locations within the normal range of visual sensitivity; black (Cluster 2) represents areas of lost function in which luminance sensitivity did not change significantly over time. Red (Cluster 3) represents a third pattern of change across time for each participant. It can be seen that, in the case of VC2 and VC3, this third pattern was an improvement from pretraining to posttraining time points. In the case of patient VC7, this third pattern represents a significantly smaller change between the two visual field tests administered than was observed in patients who underwent training (such as VC2 and VC3). Deg., Degree.

ter V1 damage is important for several reasons. First, impaired motion processing is likely responsible for most of the problems experienced by this patient population when navigating and interacting with the complex, dynamic visual environments typical of everyday life (Pambakian and Kennard, 1997; Cole, 1999; Kerkhoff, 2000; Gutteridge and McDonald, 2004; McDonald et al., 2006). Second, higher-level visual areas globally termed the MT+ complex in humans (Vaina et al., 2001) and which appear critical for learning and processing of complex visual motion in primates (Ball and Sekuler, 1982, 1987; Newsome and Paré, 1988; Dosher and Lu, 1998; Liu, 1999; Rudolph and Pasternak, 1999) are usually spared after V1 damage. Indeed, cortically blind hu- mans are thought to possess residual visual motion perception in their blind field (Riddoch, 1917; Holmes, 1918; Weiskrantz et al., 1995; Azzopardi and Cowey, 1998; Zeki and Ffytche, 1998; Azzopardi and Cowey, 2001), which can be conscious [Riddoch syndrome (Riddoch, 1917)] or unconscious [as in some types of blind sight (Weiskrantz et al., 1974)]. This residual motion perception is generally attributed to the existence of direct connections between the dLGN, the superior colliculus/pulvinar systems, and extrastriate cortex, including area MT (Cowey and Stoerig, 1991; Sincich et al., 2004).

Consistent with previous reports (Azzopardi and Cowey, 2001), our experiments showed V1-damaged patients to possess 
motion detection but poor direction discrimination abilities in their perimetrically blind field. In addition, as shown previously by Azzopardi and Cowey (2001), V1-damaged subjects in our study were completely unable to discriminate global direction of motion in their blind field. However, the novel finding emerging from the present work is that V1 damage does not eliminate the ability of the adult visual system to relearn to discriminate motion direction. In fact, our data demonstrate that damaged, adult visual systems can relearn relatively complex computations, such as the integration of motion directions and the extraction of directional signals from noise. Such learning does not occur spontaneously, although most subjects are constantly immersed in the complex, dynamic visual environment characteristic of their everyday life. Intensive and specific training is required.

\section{Pathways likely to mediate training-induced improvements in global motion discrimination after V1 damage}

Visual cortical damage in adult mammals is usually followed by some degree of spontaneous recovery. This is thought to be attributable to resolving inflammation around the lesion site, the return of function in neural circuits damaged but not destroyed by the insult, and reorganization of connections and receptive fields within surrounding cortical tissues (Eysel and Schweigart, 1999; Eysel et al., 1999). In humans with V1 damage, most spontaneous improvements in vision occur within the first few weeks after the insult (Zhang et al., 2006). They are rarely reported after the second or third month after lesion (Tiel and Kolmel, 1991; Zhang et al., 2006). Improvement in visually guided behavior can still occur during the ensuing months and years, but this generally results from the development of compensatory eye movement strategies (e.g., more numerous saccades toward blind regions of the visual field) rather than from the recovery of lost vision per se (Ishiai et al., 1987; Pambakian et al., 2000). The improvements attained in the present study were elicited long after the time frame in which spontaneous recovery is likely to occur. Thus, they cannot be explained by resolving inflammation or shortterm recovery of injured neurons.

One striking characteristic of training-induced improvements in global motion perception in the blind field of V1-damaged subjects was their tight retinotopic specificity for the trained locations. This is in contrast with the effective transfer of the (smallmagnitude) improvements in direction range thresholds observed in the same subjects' intact visual hemifields. Thus, retinotopically organized circuitry likely mediated the relearning process. Although area MT and related circuits with relatively large receptive fields (and thus looser retinotopy) appear critical for learning complex motion discrimination in an intact visual system (Lu et al., 2004; Thompson and Liu, 2006), our data suggest that such relearning in the blind field may be mediated by either different visual circuits or neurons (even MT neurons) with properties altered by the damage.

One possibility is that intact islands of V1 exist and/or that there is intact V1 at the perimeter of the brain lesions. These intact islands or strips may not function normally after the damage, hence, the "cortical blindness." However, training may stimulate and reactivate these intact pieces of V1, causing them to undergo significant plasticity, including visual map plasticity, and take over some of the lost visual functions. Learning might then exhibit V1-like specificities and the retinotopic map may appear distorted. Additional investigation is needed to assess the true functional impact of residual V1 tissue in the context of visual rehabilitation.

Nevertheless, the tight retinotopy of improvements in global motion thresholds in the blind field of V1-damaged subjects also suggests that the reverse-hierarchy model of perceptual learning (Ahissar and Hochstein, 1997) could be applied to a visual system lacking an intact V1. The principal tenet of this model is that perceptual learning occurs from higher- to lower-level visual areas, so that higher-level improvements drive learning in lowerlevel, more retinotopically organized visual areas such as V1 (Ahissar and Hochstein, 1997). Of course, one may wonder which low-level visual areas participate in this process after V1 damage. The small spatial extent of relearning in the blind field suggests that areas with relatively tight retinotopic organization such as V2 or V3 may take over some of the roles played previously by $\mathrm{V} 1$ in visual processing and learning (for functional imaging support for this notion, see the work of Ptito et al., 1999; Goebel et al., 2001).

However, although training improved direction range thresholds in the blind field, subjects also experienced improved sensitivity for the detection of brightness increments (Humphrey visual field test), improved contrast thresholds for the direction discrimination of simple, luminance-modulated sine-wave gratings, and improved motion signal thresholds. Thus, training optimized the ability of the residual visual circuitry to correctly identify simple motion directions and from a sufficient number of component dots, to extract a global directional vector. That performance improved on visual dimensions not specifically trained (but likely present in the training stimuli) suggests that global direction discrimination training may have caused an additional, general increase in visual sensitivity. Improved motion signal thresholds after training likely indicate that global direction discrimination training also increased the ability of the system to extract motion signals from noise at the retrained locations. Whether this is attributable to a decrease in the internal noise of the visual system or an increase in the efficiency of the system at processing external noise remains to be determined. Even normal task-dependent learning has been shown to be associated with a reduction in external noise and an enhancement in signal extraction (Dosher and Lu, 1998). These phenomena were interpreted to reflect plasticity in and mediation of complex motion learning by higher-level visual cortical areas such as MT rather than V1 (Dosher and Lu, 1998), an idea supported by the observation that humans show more visual learning on tasks requiring discrimination along multiple than along single perceptual dimensions (Fine and Jacobs, 2002). That learning is greater when external noise is added to an otherwise unambiguous stimulus suggests that higher-level visual areas not only mediate different aspects of visual learning than V1 but that they mediate larger learning effects as well (Fine and Jacobs, 2002). Indeed, the magnitude of learning effects in the blind field of our subjects was significantly larger than that in the intact hemifield. Thus, although the precise roles played by individual areas in relearning of complex motion perception after permanent V1 damage remains to be determined, it is likely that both lower- and higherlevel extrastriate visual cortices contribute to this process.

In conclusion, intensive, localized training on a direction integration task improved global direction discrimination in the blind field of adult humans with cortical blindness at a time when spontaneous visual improvements in the blind field are no longer thought possible (Zhang et al., 2006). Unlike perceptual learning in the intact hemifield, however, training-induced improvements in the blind field were large in magnitude, slow to attain, small in spatial extent, and retinotopically restricted to retrained locations. They were also associated with improved visual sensitivity for simpler, moving stimuli and improved ability to extract mo- 
tion signal from noise. These data provide evidence for a surprisingly large amount of perceptual plasticity in the adult visual system after damage to one of its principal components. They also demonstrate a clear benefit of repetitively forcing the damaged visual circuitry to discriminate complex, moving stimuli to recover multiple aspects of conscious visual perception. If these results continue to be supported, they offer renewed optimism for the development of effective rehabilitation strategies to treat visual deficits resulting from permanent visual cortical damage in adulthood.

\section{References}

Ahissar M, Hochstein S (1997) Task difficulty and the specificity of perceptual learning. Nature 387:401-406.

Azzopardi P, Cowey A (1998) Blindsight and visual awareness. Conscious Cogn 7:292-311.

Azzopardi P, Cowey A (2001) Motion discrimination in cortically blind patients. Brain 124:30-46.

Bach-y-Rita P (1983) Controlling variables eliminates hemianopia rehabilitation results. Behav Brain Sci 6:448.

Ball K, Sekuler R (1982) A specific and enduring improvement in visual motion discrimination. Science 218:697-698.

Ball K, Sekuler R (1987) Direction-specific improvement in motion discrimination. Vision Res 27:953-965.

Balliet R, Blood KM, Bach-y-Rita P (1985) Visual field rehabilitation in the cortically blind? J Neurol Neurosurg Psychiatr 48:1113-1124.

Barbur JL, Harlow AJ, Weiskrantz L (1994) Spatial and temporal response properties of residual vision in a case of hemianopia. Philos Trans R Soc Lond B Biol Sci 343:157-166.

Bartels A, Zeki S (2000) The architecture of the color center in the human visual brain: new results and a review. Eur J Neurosci 12:172-193.

Cole M (1999) When the left brain is not right the right brain may be left: report of personal experience of occipital hemianopia. J Neurol Neurosurg Psychiatr 67:169-173.

Cowey A, Stoerig P (1991) The neurobiology of blindsight. Trends Neurosci 14:140-145.

Crist RE, Kapadia MK, Westheimer G, Gilbert CD (1997) Perceptual learning of spatial localization: specificity for orientation, position, and context. J Neurophysiol 78:2889-2894.

Crist RE, Li W, Gilbert CD (2001) Learning to see: experience and attention in primary visual cortex. Nat Neurosci 4:519-525.

Damasio AR, Damasio H, Van Hoesen GW (1982) Prosopagnosia: anatomic basis and behavioral mechanisms. Neurology 32:331-341.

Dosher BA, Lu ZL (1998) Perceptual learning reflects external noise filtering and internal noise reduction through channel reweighting. Proc Natl Acad Sci U S A 95:13988-13993.

Duda RO, Hart PE (1973) Pattern classification and scene analysis, pp 189 225. New York: Wiley.

Eysel UT, Schweigart G (1999) Increased receptive field size in the surround of chronic lesions in the adult cat visual cortex. Cereb Cortex 9:101-109.

Eysel UT, Schweigart G, Mittmann T, Eyding D, Qu Y, Vandesande F, Orban G, Arckens L (1999) Reorganization in the visual cortex after retinal and cortical damage. Restor Neurol Neurosci 15:153-164.

Felleman DJ, Van Essen DC (1991) Distributed hierarchical processing in the primate cerebral cortex. Cereb Cortex 1:1-47.

Fine I, Jacobs RA (2002) Comparing perceptual learning across tasks: a review. J Vis 2:190-203.

Fiorentini A, Berardi N (1980) Perceptual learning specific for orientation and spatial frequency. Nature 287:43-44.

Furmanski CS, Schluppeck D, Engel SA (2004) Learning strengthens the response of primary visual cortex to simple patterns. Curr Biol 14:573-578.

Goebel R, Muckli L, Zanella FE, Singer W, Stoerig P (2001) Sustained extrastriate cortical activation without visual awareness revealed by fMRI studies in hemianopic patients. Vision Res 41:1459-1474.

Goodale MA (1998) Visuomotor control: where does vision end and action begin? Curr Biol 8:R489-R491.

Gutteridge IF, McDonald RA (2004) Hemianopic visual field loss as the first clinical evidence of occipital arteriovenous malformation. Clin Exp Optom 87:394-399.

Hallett M (2001) Plasticity of the human motor cortex and recovery from stroke. Brain Res Rev 36:169-174.
Holmes G (1918) Disturbances of vision by cerebral lesions. Br J Ophthalmol 2:353-384.

Huxlin KR, Pasternak T (2004) Training-induced recovery of visual motion perception after extrastriate cortical damage in the adult cat. Cereb Cortex 14:81-90.

Huxlin KR, Williams JM, Price T (2008) A neurochemical signature of visual recovery after extrastriate cortical damage in the adult cat. J Comp Neurol 508:45-61.

Ishiai S, Furukawa T, Tsukagoshi H (1987) Eye-fixation patterns in homonymous hemianopia and unilateral spatial neglect. Neuropsychologia 25:675-679.

Karni A, Sagi D (1991) Where practice makes perfect in texture discrimination: evidence for primary visual cortex plasticity. Proc Natl Acad Sci U S A 88:4966-4970.

Karni A, Sagi D (1993) The time course of learning a visual skill. Nature 365:250-252.

Kasten E, Wüst S, Behrens-Baumann W, Sabel BA (1998) Computer-based training for the treatment of partial blindness. Nat Med 4:1083-1087.

Kerkhoff G (2000) Neurovisual rehabiliation: recent developments and future directions. J Neurol Neurosurg Psychiatr 68:691-706.

Liu Z (1999) Perceptual learning in motion discrimination that generalizes across motion directions. Proc Natl Acad Sci U S A 96:14085-14087.

Lu H, Qian N, Liu Z (2004) Learning motion discrimination with suppressed MT. Vision Res 44:1817-1825.

Maunsell JH, Newsome WT (1987) Visual processing in monkey extrastriate cortex. Annu Rev Neurosci 10:363-401.

McDonald SA, Spitsyna G, Shillcock RC, Wise RJ, Leff AP (2006) Patients with hemianopic alexia adopt an inefficient eye movement strategy when reading text. Brain 129:158-167.

Newsome WT, Paré EB (1988) A selective impairment of motion perception following lesions of the middle temporal visual area (MT). J Neurosci 8:2201-2211.

Pambakian AL, Kennard C (1997) Can visual function be restored in patients with homonymous hemianopia. Br J Ophthalmol 81:324-328.

Pambakian AL, Wooding DS, Patel N, Morland AB, Kennard C, Mannan SK (2000) Scanning the visual world: a study of patients with homonymous hemianopia. J Neurol Neurosurg Psychiatr 69:751-759.

Pourtois G, Rauss KS, Vuilleumier P, Schwartz S (2008) Effects of perceptual learning on primary visual cortex activity in humans. Vision Res 48:55-62.

Ptito M, Johannsen P, Faubert J, Gjedde A (1999) Activation of human extrageniculostriate pathways after damage to area V1. Neuroimage 9:97-107.

Ramachandran VS, Braddick O (1973) Orientation-specific learning in stereopsis. Perception 2:371-376.

Raninen A, Vanni S, Hyvärinen L, Näsänen R (2007) Temporal sensitivity in a hemianopic visual field can be improved by long-term training using flicker stimulation. J Neurol Neurosurg Psychiatr 78:66-73.

Reinhard J, Schreiber A, Schiefer U, Kasten E, Sabel BA, Kenkel S, Vonthein R, Trauzettel-Klosinski S (2005) Does visual restitution training change absolute homonymous visual field defects? A fundus controlled study. Br J Ophthalmol 89:30-35.

Riddoch G (1917) Dissociations of visual perceptions due to occipital injuries, with especial reference to appreciation of movement. Brain 40:15-57.

Rudolph K, Pasternak T (1999) Transient and permanent deficits in motion perception after lesions of cortical areas MT and MST in the macaque monkey. Cereb Cortex 9:90-100.

Sahraie A, Trevethan CT, Weiskrantz L, Olson J, MacLeod MJ, Murray AD, Dijkhuizen RS, Counsell C, Coleman R (2003) Spatial channels of visual processing in cortical blindness. Eur J Neurosci 18:1189-1196.

Sahraie A, Trevethan CT, MacLeod MJ, Murray AD, Olson JA, Weiskrantz L (2006) Increased sensitivity after repeated stimulation of residual spatial channels in blindsight. Proc Natl Acad Sci U S A 103:14971-14976.

Schoups A, Vogels R, Qian N, Orban GA (2001) Practising orientation identification improves orientation coding in V1 neurons. Nature 412:549-553.

Schwartz S, Maquet P, Frith C (2002) Neural correlates of perceptual learning: a functional MRI study of visual texture discrimination. Proc Natl Acad Sci U S A 99:17137-17142.

Shiu LP, Pashler H (1992) Improvement in line orientation discrimination is retinally local but dependent on cognitive set. Percept Psychophys $52: 582-588$. 
Sincich LC, Park KF, Wohlgemuth MJ, Horton JC (2004) Bypassing V1: a direct genicular input for area MT. Nat Neurosci 7:1123-1128.

Taub E, Uswatte G, Elbert T (2002) New treatments in neurorehabilitation founded on basic research. Nat Rev Neurosci 3:228-236.

Thompson B, Liu Z (2006) Learning motion discrimination with suppressed and un-suppressed MT. Vision Res 46:2110-2121.

Tiel K, Kolmel HW (1991) Patterns of recovery from homonymous hemianopia subsequent to infarction in the distribution of the posterior cerebral artery. Neuro-ophthalmology 11:33-39.

Vaina LM (1994) Functional segregation of color and motion processing in the human visual cortex: clinical evidence. Cereb Cortex 4:555-572.

Vaina LM, Sundareswaran V, Harris JG (1995) Learning to ignore: psychophysics and computational modeling of fast learning of direction in noisy motion stimuli. Brain Res Cogn Brain Res 2:155-163.

Vaina LM, Cowey A, Eskew RT Jr, LeMay M, Kemper T (2001) Regional cerebral correlates of global motion perception. Evidence from unilateral cerebral brain damage. Brain 124:310-321.
Walker MP, Stickgold R, Jolesz FA, Yoo SS (2005) The functional anatomy of sleep-dependent visual skill learning. Cereb Cortex 15:1666-1675.

Weiskrantz L, Warrington EK, Sanders MD, Marshall J (1974) Visual capacity in the hemianopic field following a restricted occipital ablation. Brain 97:709-728.

Weiskrantz L, Barbur JL, Sahraie A (1995) Parameters affecting conscious versus unconscious visual discrimination with damage to the visual cortex (v1). Proc Natl Acad Sci U S A 92:6122-6126.

Zeki S (1991) Cerebral akinetopsia (visual motion blindness). A review. Brain 114:811-824.

Zeki S, Ffytche DH (1998) The Riddoch syndrome: insights into the neurobiology of conscious vision. Brain 121:25-45.

Zhang X, Kedar S, Lynn MJ, Newman NJ, Biousse V (2006) Natural history of homonymous hemianopia. Neurology 66:901-905.

Zihl J (1981) Recovery of visual functions in patients with cerebral blindness: effect of specific practice with saccadic localization. Exp Brain Res 44:159-169. 